\title{
On The Bounded Oscillation of Certain Second-order Nonlinear Neutral Delay Dynamic Equations with Oscillating Coefficients
}

\author{
Da-Xue Chen (Corresponding author) \\ College of Science, Hunan Institute of Engineering \\ 88 East Fuxing Road, Xiangtan 411104, Hunan, China \\ E-mail: cdx2003@163.com \\ Guang-Hui Liu \\ College of Science, Hunan Institute of Engineering \\ 88 East Fuxing Road, Xiangtan 411104, Hunan, China \\ E-mail: lgh233@163.com
}

Received: November 24, 2010 Accepted: December 10, 2010 doi:10.5539/jmr.v3n2p193

This work was supported by the Science and Technology Program of Hunan Province of P. R. China (Grant No. 2010FJ6021).

\section{Abstract}

We investigate the bounded oscillation of the second-order nonlinear neutral delay dynamic equation with oscillating coefficients

$$
\left(r(t)\left|[x(t)+p(t) x(\tau(t))]^{\Delta}\right|^{\alpha-1}[x(t)+p(t) x(\tau(t))]^{\Delta}\right)^{\Delta}+q(t)|x(t)|^{\beta-1} x(t)=0
$$

on an arbitrary time scale $\mathbb{T}$, where $p$ is an oscillating function defined on $\mathbb{T}$ and $\alpha, \beta>0$ are constants, and obtain several sufficient conditions for the oscillation of all bounded solutions of the equation when $\beta>\alpha, \beta=\alpha$ and $\beta<\alpha$, respectively. Our results extend and complement some known results where $p(t) \equiv 0$ and $\alpha, \beta$ are quotients of odd positive integers.

Keywords: Bounded oscillation, Neutral delay dynamic equation, Oscillating coefficient

\section{Introduction}

In the last decade, the study of dynamic equations on time scales has been a very active area of research and there has been much research activity concerning the oscillation and nonoscillation of solutions of various dynamic equations on time scales, and we refer the reader to (Agarwal et al., 2003; Chen \& Liu, 2008; Chen, 2010; Erbe et al., 2007; Grace et al., 2008; Grace et al., 2009; Han et al., 2007; Hassan, 2008; Medico \& Kong, 2004; Saker, 2005) and the references cited therein. Saker (2005) established some oscillation criteria for the second-order half-linear dynamic equation

$$
\left(r(t)\left(x^{\Delta}(t)\right)^{\alpha}\right)^{\Delta}+q(t) x^{\alpha}(t)=0
$$

on time scales, where $\alpha>1$ is an odd positive integer, and $r$ and $q$ are positive rd-continuous functions.

Hassan (2008) considered the same Equation (1), where $\alpha$ is a quotient of odd positive integers, and obtained some sufficient conditions for the oscillation. Hassan (2008) improved and extended the results of Saker (2005).

Recently, Grace et al. (2008, 2009) studied the oscillation of the second-order nonlinear dynamic equation

$$
\left(r(t)\left(x^{\Delta}(t)\right)^{\alpha}\right)^{\Delta}+q(t) x^{\beta}(t)=0
$$

on time scales, where $\alpha, \beta$ are quotients of odd positive integers, and $r$ and $q$ are positive rd-continuous functions. Grace et al. (2008, 2009) gave some new oscillation results for (2) when $\beta>\alpha, \beta=\alpha$ and $\beta<\alpha$, respectively.

Following the above-mentioned research trend, in this paper we consider the oscillation of all bounded solutions of the nonlinear second-order neutral delay dynamic equation with oscillating coefficients

$$
\left(r(t)\left|[x(t)+p(t) x(\tau(t))]^{\Delta}\right|^{\alpha-1}[x(t)+p(t) x(\tau(t))]^{\Delta}\right)^{\Delta}+q(t)|x(t)|^{\beta-1} x(t)=0
$$

on an arbitrary time scale $\mathbb{T}$. Here, and in what follows, the following conditions are assumed to hold: $p$ is an oscillating function defined on $\mathbb{T}, \lim _{t \rightarrow \infty} p(t)=0, \alpha, \beta>0$ are constants, $r$ and $q$ are positive rd-continuous functions on time scale interval $\left[t_{0}, \infty\right)$, and $\tau: \mathbb{T} \rightarrow \mathbb{T}$ satisfies $\lim _{t \rightarrow \infty} \tau(t)=\infty$. Since the oscillatory behavior of solutions near infinity is our primary concern, we make the assumption that $\sup \mathbb{T}=\infty$. 
It is easy to see that (1) and (2) are special cases of (3), and all the results of Saker (2005), Hassan (2008) and Grace et al. $(2008,2009)$ can not be applied to (3) when $p(t) \not \equiv 0$ or $\alpha, \beta$ are not equal to quotients of odd positive integers. Therefore, it is of great interest to study the oscillation of (3) when $p$ is an oscillating function and $\alpha, \beta>0$ are constants. The purpose of this paper is to establish some new oscillation criteria for (3). Our results extend and complement the results of Saker (2005), Hassan (2008) and Grace et al. (2008, 2009).

Recall that a solution of (3) is a nontrivial real function $x$ such that $x(t)+p(t) x(\tau(t)) \in C_{r d}^{1}\left[t_{x}, \infty\right)$ and $r(t) \mid[x(t)+$ $p(t) x(\tau(t))]\left.^{\Delta}\right|^{\alpha-1}[x(t)+p(t) x(\tau(t))]^{\Delta} \in C_{r d}^{1}\left[t_{x}, \infty\right)$ for a certain $t_{x} \geq t_{0}$ and satisfying (3) for $t \geq t_{x}$. Our attention is restricted to those solutions of (3) which exist on the half-line $\left[t_{x}, \infty\right)$ and satisfy $\sup \left\{|x(t)|: t>t_{*}\right\}>0$ for any $t_{*} \geq t_{x}$. A solution $x$ of (3) is said to be oscillatory if it is neither eventually positive nor eventually negative. Otherwise it is nonoscillatory. Equation (3) is said to be oscillatory if all its solutions are oscillatory.

In what follows, for convenience, when we write a functional inequality without specifying its domain of validity we assume that it holds for all sufficiently large $t$.

\section{Some preliminaries on time scales}

For completeness, we recall the following concepts related to the notion of time scales. More details can be found in (Bohner \& Peterson, 2001; Bohner \& Peterson, 2003).

A time scale $\mathbb{T}$ is an arbitrary nonempty closed subset of the real numbers $\mathbb{R}$. We assume throughout that $\mathbb{T}$ has the topology that it inherits from the standard topology on the real numbers $\mathbb{R}$. Some examples of time scales are as follows: the real numbers $\mathbb{R}$, the integers $\mathbb{Z}$, the positive integers $\mathbb{N}$, the nonnegative integers $\mathbb{N}_{0},[0,1] \cup[2,3],[0,1] \cup \mathbb{N}, h \mathbb{Z}:=$ $\{h k: k \in \mathbb{Z}, h>0\}$ and $\overline{q^{\mathbb{Z}}}:=\left\{q^{k}: k \in \mathbb{Z}, q>1\right\} \cup\{0\}$. But the rational numbers $\mathbb{Q}$, the complex numbers $\mathbb{C}$ and the open interval $(0,1)$ are no time scales. Many other interesting time scales exist, and they give rise to plenty of applications (see (Bohner \& Peterson, 2001)).

For $t \in \mathbb{T}$, the forward jump operator and the backward jump operator are defined by:

$$
\sigma(t):=\inf \{s \in \mathbb{T}: s>t\}, \quad \rho(t):=\sup \{s \in \mathbb{T}: s<t\},
$$

where $\inf \varnothing=\sup \mathbb{T}$ (i.e., $\sigma(t)=t$ if $\mathbb{T}$ has a maximum $t$ ) and $\sup \varnothing=\inf \mathbb{T}$ (i.e., $\rho(t)=t$ if $\mathbb{T}$ has a minimum $t$ ), here $\varnothing$ denotes the empty set.

Let $t \in \mathbb{T}$. If $\sigma(t)>t$, we say that $t$ is right-scattered, while if $\rho(t)<t$, we say that $t$ is left-scattered. Points that are right-scattered and left-scattered at the same time are called isolated. Also, if $t<\sup \mathbb{T}$ and $\sigma(t)=t$, then $t$ is called right-dense, and if $t>\inf \mathbb{T}$ and $\rho(t)=t$, then $t$ is called left-dense. The graininess function $\mu: \mathbb{T} \rightarrow[0, \infty)$ is defined by

$$
\mu(t):=\sigma(t)-t
$$

We also need below the set $\mathbb{T}^{\kappa}$ : If $\mathbb{T}$ has a left-scattered maximum $m$, then $\mathbb{T}^{\kappa}=\mathbb{T}-\{m\}$. Otherwise, $\mathbb{T}^{\kappa}=\mathbb{T}$. Let $f: \mathbb{T} \rightarrow \mathbb{R}$, then we define the function $f^{\sigma}: \mathbb{T}^{\kappa} \rightarrow \mathbb{R}$ by

$$
f^{\sigma}(t):=f(\sigma(t)) \quad \text { for all } t \in \mathbb{T}^{\kappa},
$$

i.e., $f^{\sigma}:=f \circ \sigma$.

For $a, b \in \mathbb{T}$ with $a<b$, we define the interval $[a, b]$ in $\mathbb{T}$ by

$$
[a, b]:=\{t \in \mathbb{T}: a \leq t \leq b\} .
$$

Open intervals and half-open intervals, etc. are defined accordingly.

Fix $t \in \mathbb{T}^{\kappa}$ and let $f: \mathbb{T} \rightarrow \mathbb{R}$. Define $f^{\Delta}(t)$ to be the number (provided it exists) with the property that given any $\varepsilon>0$, there is a neighbourhood $U$ of $t$ such that

$$
\left|[f(\sigma(t))-f(s)]-f^{\Delta}(t)[\sigma(t)-s]\right| \leq \varepsilon|\sigma(t)-s| \quad \text { for all } s \in U .
$$

In this case, we say that $f^{\Delta}(t)$ is the (delta) derivative of $f$ at $t$ and that $f$ is (delta) differentiable at $t$.

Assume that $f: \mathbb{T} \rightarrow \mathbb{R}$ and let $t \in \mathbb{T}^{\kappa}$. If $f$ is (delta) differentiable at $t$, then

$$
f(\sigma(t))=f(t)+\mu(t) f^{\Delta}(t) .
$$

A function $f: \mathbb{T} \rightarrow \mathbb{R}$ is said to be right-dense continuous (rd-continuous) provided it is continuous at each right-dense point in $\mathbb{T}$ and its left-sided limits exist (finite) at all left-dense points in $\mathbb{T}$. The set of all such rd-continuous functions is denoted by

$$
C_{r d}(\mathbb{T})=C_{r d}(\mathbb{T}, \mathbb{R})
$$


The set of functions $f: \mathbb{T} \rightarrow \mathbb{R}$ that are (delta) differentiable and whose (delta) derivative is rd-continuous is denoted by

$$
C_{r d}^{1}(\mathbb{T})=C_{r d}^{1}(\mathbb{T}, \mathbb{R}) .
$$

We will make use of the following product and quotient rules for the (delta) derivative of the product $f g$ and the quotient $f / g$ of two (delta) differentiable functions $f$ and $g$ :

$$
(f g)^{\Delta}=f^{\Delta} g+f^{\sigma} g^{\Delta}=f g^{\Delta}+f^{\Delta} g^{\sigma}
$$

and

$$
\left(\frac{f}{g}\right)^{\Delta}=\frac{f^{\Delta} g-f g^{\Delta}}{g g^{\sigma}}
$$

where $g^{\sigma}=g \circ \sigma$ and $g g^{\sigma} \neq 0$.

For $a, b \in \mathbb{T}$ and a (delta) differentiable function $f$, the Cauchy (delta) integral of $f^{\Delta}$ is defined by

$$
\int_{a}^{b} f^{\Delta}(t) \Delta t=f(b)-f(a) \text {. }
$$

The integration by parts formula reads

$$
\int_{a}^{b} f(t) g^{\Delta}(t) \Delta t=f(b) g(b)-f(a) g(a)-\int_{a}^{b} f^{\Delta}(t) g^{\sigma}(t) \Delta t
$$

or

$$
\int_{a}^{b} f^{\sigma}(t) g^{\Delta}(t) \Delta t=f(b) g(b)-f(a) g(a)-\int_{a}^{b} f^{\Delta}(t) g(t) \Delta t .
$$

The infinite integral is defined as

$$
\int_{a}^{\infty} f(s) \Delta s=\lim _{t \rightarrow \infty} \int_{a}^{t} f(s) \Delta s .
$$

Lemma 1. ((Bohner \& Peterson, 2001), p. 32, Theorem 1.87) Let $f: \mathbb{R} \rightarrow \mathbb{R}$ be continuously differentiable and suppose $g: \mathbb{T} \rightarrow \mathbb{R}$ is delta differentiable. Then $f \circ g: \mathbb{T} \rightarrow \mathbb{R}$ is delta differentiable and satisfies

$$
(f \circ g)^{\Delta}(t)=\left\{\int_{0}^{1} f^{\prime}\left(g(t)+h \mu(t) g^{\Delta}(t)\right) d h\right\} g^{\Delta}(t) .
$$

\section{Main results}

Theorem 1. Let $\beta>\alpha$, and suppose that the following conditions hold:

$$
\begin{gathered}
\int_{t_{0}}^{\infty}\left(\frac{1}{r(t)}\right)^{1 / \alpha} \Delta t=\infty, \\
\int_{t}^{\infty} q(s) \Delta s<\infty \quad \text { for } \quad t \in\left[t_{0}, \infty\right),
\end{gathered}
$$

and

$$
\int_{t_{0}}^{\infty} r^{-\frac{1}{\alpha}}(s) H^{\sigma}(s, c) \Delta s=\infty
$$

where $H(t, c):=\left[Q(t)+c \int_{t}^{\infty} r^{-\frac{1}{\alpha}}(s)\left(Q^{\sigma}(s)\right)^{(1+\alpha) / \alpha} \Delta s\right]^{1 / \alpha}$, here $c$ is an arbitrary positive constant, $Q(t):=\varepsilon^{\beta} \int_{t}^{\infty} q(s) \Delta s$ and $\varepsilon \in(0,1)$ is an arbitrary constant. Then every bounded solution of (3) is oscillatory.

Proof. Suppose that $x$ is a bounded nonoscillatory solution of (3). Without loss of generality, we may assume that $x$ is a bounded eventually positive solution of (3). Then there exists $t_{1} \in\left[t_{0}, \infty\right)$ such that

$$
x(t)>0, \quad t \in\left[t_{1}, \infty\right) .
$$

Let

$$
y(t):=x(t)+p(t) x(\tau(t)), \quad t \in \mathbb{T} .
$$


It is easy to see that $y$ is bounded. It follows from (3), (13) and (12) that

$$
\left(r(t)\left|y^{\Delta}(t)\right|^{\alpha-1} y^{\Delta}(t)\right)^{\Delta}=-q(t) x^{\beta}(t)<0, \quad t \in\left[t_{1}, \infty\right) .
$$

Thus, $r(t)\left|y^{\Delta}(t)\right|^{\alpha-1} y^{\Delta}(t)$ is strictly decreasing on $\left[t_{1}, \infty\right)$ and is eventually of one sign. We claim

$$
y^{\Delta}(t)>0, \quad t \in\left[t_{1}, \infty\right) .
$$

Assume on the contrary, then there exists $t_{2} \in\left[t_{1}, \infty\right)$ such that $y^{\Delta}\left(t_{2}\right) \leq 0$. Take $t_{3}>t_{2}$. Since $r(t)\left|y^{\Delta}(t)\right|^{\alpha-1} y^{\Delta}(t)$ is strictly decreasing on $\left[t_{1}, \infty\right)$, we have

$$
r(t)\left|y^{\Delta}(t)\right|^{\alpha-1} y^{\Delta}(t) \leq r\left(t_{3}\right)\left|y^{\Delta}\left(t_{3}\right)\right|^{\alpha-1} y^{\Delta}\left(t_{3}\right):=M<r\left(t_{2}\right)\left|y^{\Delta}\left(t_{2}\right)\right|^{\alpha-1} y^{\Delta}\left(t_{2}\right) \leq 0
$$

for $t \in\left[t_{3}, \infty\right)$. Thus, we obtain $y^{\Delta}(t) \leq-(-M)^{\frac{1}{\alpha}}\left(\frac{1}{r(t)}\right)^{1 / \alpha}$ for $t \in\left[t_{3}, \infty\right)$. Integrating both sides of the last inequality from $t_{3}$ to $t$, we get

$$
y(t)-y\left(t_{3}\right) \leq-(-M)^{\frac{1}{\alpha}} \int_{t_{3}}^{t}\left(\frac{1}{r(s)}\right)^{1 / \alpha} \Delta s, \quad t \in\left[t_{3}, \infty\right) .
$$

Letting $t \rightarrow \infty$ and noticing (9), we see that $\lim _{t \rightarrow \infty} y(t)=-\infty$. This contradicts the fact that $y$ is bounded. Hence, (15) holds. From (15) we find that $y(t)$ is strictly increasing on $\left[t_{1}, \infty\right)$ and is eventually of one sign. We now claim that $y(t)$ is eventually positive, i.e., there exists $t_{4} \in\left[t_{1}, \infty\right)$ such that

$$
y(t)>0, \quad t \in\left[t_{4}, \infty\right)
$$

Assume on the contrary, then $y(t)$ is eventually nonpositive and there exists $t_{5} \in\left[t_{1}, \infty\right)$ such that $y(t) \leq 0$ for $t \in\left[t_{5}, \infty\right)$. Therefore, from (13) and (12) we conclude

$$
p(t) x(\tau(t))=y(t)-x(t)<0 .
$$

Since $p$ is an oscillating function on $\mathbb{T}$ and $x(\tau(t))>0$, we find a contradiction to (17). Thus, (16) holds. From (15), (16) and the property that $y$ is bounded, we get $\lim _{t \rightarrow \infty} y(t):=L>0$. Hence, from (13) we have $x(t)=y(t)-p(t) x(\tau(t))>\varepsilon y(t)$ for $\varepsilon \in(0,1)$. In view of (14) and (15), there exists $t_{6} \in\left[t_{4}, \infty\right)$ such that

$$
\left[r(t)\left(y^{\Delta}(t)\right)^{\alpha}\right]^{\Delta} \leq-\varepsilon^{\beta} q(t) y^{\beta}(t), \quad t \in\left[t_{6}, \infty\right) .
$$

Define the function $w$ by

$$
w(t)=\frac{r(t)\left(y^{\Delta}(t)\right)^{\alpha}}{y^{\beta}(t)}, \quad t \in\left[t_{6}, \infty\right) .
$$

It is easy to see that $w(t)>0$ for $t \in\left[t_{6}, \infty\right)$. By the product and quotient rules (5) and (6) for the delta derivative and then from (18) and (19), we get

$$
\begin{aligned}
w^{\Delta} & =\left(r\left(y^{\Delta}\right)^{\alpha}\right)^{\Delta} \frac{1}{y^{\beta}}+\left(r\left(y^{\Delta}\right)^{\alpha}\right)^{\sigma}\left(\frac{1}{y^{\beta}}\right)^{\Delta} \\
& \leq-q \varepsilon^{\beta}-\left(r\left(y^{\Delta}\right)^{\alpha}\right)^{\sigma} \frac{\left(y^{\beta}\right)^{\Delta}}{y^{\beta}\left(y^{\beta}\right)^{\sigma}} \\
& =-q \varepsilon^{\beta}-w^{\sigma} \frac{\left(y^{\beta}\right)^{\Delta}}{y^{\beta}} \text { on }\left[t_{6}, \infty\right) .
\end{aligned}
$$

By Lemma 1, for $t \in\left[t_{6}, \infty\right)$ we obtain

$$
\begin{aligned}
\left(y^{\beta}(t)\right)^{\Delta} & =\beta\left\{\int_{0}^{1}\left[y(t)+h \mu(t) y^{\Delta}(t)\right]^{\beta-1} d h\right\} y^{\Delta}(t) \\
& =\beta\left\{\int_{0}^{1}\left[(1-h) y(t)+h y^{\sigma}(t)\right]^{\beta-1} d h\right\} y^{\Delta}(t) \\
& \geq \begin{cases}\beta(y(t))^{\beta-1} y^{\Delta}(t), & \beta>1, \\
\beta\left(y^{\sigma}(t)\right)^{\beta-1} y^{\Delta}(t), & 0<\beta \leq 1 .\end{cases}
\end{aligned}
$$

Thus, on $\left[t_{6}, \infty\right)$ we have

$$
\frac{\left(y^{\beta}\right)^{\Delta}}{y^{\beta}} \geq \begin{cases}\beta \frac{y^{\Delta}}{y}, & \beta>1, \\ \beta \frac{\left(y^{\sigma}\right)^{\beta-1}}{y^{\beta}} y^{\Delta}, & 0<\beta \leq 1 .\end{cases}
$$


Noticing the fact that $y$ is an increasing function on $\left[t_{6}, \infty\right)$ and $t \leq \sigma(t)$, we get $y(t) \leq y^{\sigma}(t)$ for $t \in\left[t_{6}, \infty\right)$. Therefore, it follows from (21) that

$$
\frac{\left(y^{\beta}\right)^{\Delta}}{y^{\beta}} \geq \beta \frac{y^{\Delta}}{y^{\sigma}} \quad \text { on }\left[t_{6}, \infty\right) \text { for } \beta>0 .
$$

Using (22) in (20), we obtain

$$
w^{\Delta} \leq-q \varepsilon^{\beta}-\beta w^{\sigma} \frac{y^{\Delta}}{y^{\sigma}} \quad \text { on }\left[t_{6}, \infty\right)
$$

Since $r^{\frac{1}{\alpha}} y^{\Delta}$ is a decreasing function on $\left[t_{6}, \infty\right)$ and $t \leq \sigma(t)$, we conclude $r^{\frac{1}{\alpha}} y^{\Delta} \geq\left(r^{\frac{1}{\alpha}} y^{\Delta}\right)^{\sigma}$ on $\left[t_{6}, \infty\right)$. Hence, from (19) we obtain

$$
y^{\Delta} \geq r^{-\frac{1}{\alpha}}\left(w^{\sigma}\right)^{\frac{1}{\alpha}}\left(y^{\sigma}\right)^{\frac{\beta}{\alpha}} \quad \text { on }\left[t_{6}, \infty\right) .
$$

Substituting (24) in (23), we have

$$
w^{\Delta} \leq-q \varepsilon^{\beta}-\beta r^{-\frac{1}{\alpha}}\left(w^{\sigma}\right)^{1+\frac{1}{\alpha}}\left(y^{\sigma}\right)^{\frac{\beta}{\alpha}-1} \quad \text { on }\left[t_{6}, \infty\right) .
$$

Integrating both sides of the last inequality from $t$ to $u\left(u \geq t \geq t_{6}\right)$ and letting $u \rightarrow \infty$, we obtain

$$
w(t) \geq \varepsilon^{\beta} \int_{t}^{\infty} q(s) \Delta s+\beta \int_{t}^{\infty} r^{-\frac{1}{\alpha}}(s)\left(w^{\sigma}(s)\right)^{1+\frac{1}{\alpha}}\left(y^{\sigma}(s)\right)^{\frac{\beta}{\alpha}-1} \Delta s, \quad t \in\left[t_{6}, \infty\right) .
$$

It is clear that $w(t) \geq Q(t):=\varepsilon^{\beta} \int_{t}^{\infty} q(s) \Delta s$ for $t \geq t_{6}$. Thus, we get

$$
w(t) \geq Q(t)+\beta \int_{t}^{\infty} r^{-\frac{1}{\alpha}}(s)\left(Q^{\sigma}(s)\right)^{1+\frac{1}{\alpha}}\left(y^{\sigma}(s)\right)^{\frac{\beta}{\alpha}-1} \Delta s, \quad t \in\left[t_{6}, \infty\right) .
$$

Since $\beta>\alpha$ and $y$ is an increasing function on $\left[t_{6}, \infty\right)$, there exist a $t_{7} \geq t_{6}$ and a positive constant $c_{1}$ such that

$$
\left(y^{\sigma}(s)\right)^{\frac{\beta}{\alpha}-1} \geq c_{1}, \quad s \in\left[t_{7}, \infty\right) .
$$

Using (26) in (25), we see

$$
w(t) \geq Q(t)+\beta c_{1} \int_{t}^{\infty} r^{-\frac{1}{\alpha}}(s)\left(Q^{\sigma}(s)\right)^{1+\frac{1}{\alpha}} \Delta s:=H^{\alpha}(t, c), \quad t \in\left[t_{7}, \infty\right),
$$

where $c:=\beta c_{1}$. Since $r\left(y^{\Delta}\right)^{\alpha}$ is decreasing on $\left[t_{7}, \infty\right)$ and $t \leq \sigma(t)$, we have $r\left(y^{\Delta}\right)^{\alpha} \geq\left(r\left(y^{\Delta}\right)^{\alpha}\right)^{\sigma}$ on $\left[t_{7}, \infty\right)$. Therefore, we obtain

which implies

$$
\frac{r\left(y^{\Delta}\right)^{\alpha}}{\left(y^{\sigma}\right)^{\beta}} \geq \frac{\left(r\left(y^{\Delta}\right)^{\alpha}\right)^{\sigma}}{\left(y^{\sigma}\right)^{\beta}}=w^{\sigma} \geq\left(H^{\alpha}(t, c)\right)^{\sigma} \quad \text { on }\left[t_{7}, \infty\right)
$$

$$
\left(y^{\sigma}\right)^{-\delta} y^{\Delta} \geq r^{-\frac{1}{\alpha}} H^{\sigma}(t, c) \text { on }\left[t_{7}, \infty\right),
$$

where $\delta:=\beta / \alpha>1$. Applying Lemma 1 , we get

$$
\begin{aligned}
\left(y^{1-\delta}\right)^{\Delta}(t) & =(1-\delta)\left\{\int_{0}^{1}\left[y(t)+h \mu(t) y^{\Delta}(t)\right]^{-\delta} d h\right\} y^{\Delta}(t) \\
& =(1-\delta)\left\{\int_{0}^{1}\left[(1-h) y(t)+h y^{\sigma}(t)\right]^{-\delta} d h\right\} y^{\Delta}(t) \\
& \leq(1-\delta)\left(y^{\sigma}(t)\right)^{-\delta} y^{\Delta}(t) \quad \text { on }\left[t_{7}, \infty\right) .
\end{aligned}
$$

Thus, we obtain

$$
\frac{\left(y^{1-\delta}\right)^{\Delta}}{1-\delta} \geq\left(y^{\sigma}\right)^{-\delta} y^{\Delta} \quad \text { on }\left[t_{7}, \infty\right)
$$

From (27) and (28), we conclude

$$
\left(y^{1-\delta}\right)^{\Delta} /(1-\delta) \geq r^{-\frac{1}{\alpha}} H^{\sigma}(t, c) \text { on }\left[t_{7}, \infty\right) .
$$

Integrating both sides of the last inequality from $t_{7}$ to $t\left(t \geq t_{7}\right)$, we have

$$
\int_{t_{7}}^{t} r^{-\frac{1}{\alpha}}(s) H^{\sigma}(s, c) \Delta s \leq \frac{\left(y^{1-\delta}\right)\left(t_{7}\right)}{\delta-1} .
$$

Letting $t \rightarrow \infty$, we see $\int_{t_{5}}^{\infty} r^{-\frac{1}{\alpha}}(s) H^{\sigma}(s, c) \Delta s \leq \frac{\left(y^{1-\delta}\right)\left(t_{5}\right)}{\delta-1}<\infty$, which contradicts (11). Hence, the proof is complete. 
Theorem 2. Let $\beta=\alpha$, and suppose that (9) and (10) hold. If

$$
\limsup _{t \rightarrow \infty}\left(\int_{t_{0}}^{t} r^{-\frac{1}{\alpha}}(s) \Delta s\right) H(t, \alpha)>1,
$$

where $H$ is defined as in Theorem 1, then all bounded solutions of (3) are oscillatory.

Proof. Assume that $x$ is a bounded nonoscillatory solution of (3). Without loss of generality, we may assume that $x$ is a bounded eventually positive solution of (3). Proceeding as in the proof of Theorem 1, we find that (25) takes the form

$$
\begin{aligned}
w(t) & \geq Q(t)+\alpha \int_{t}^{\infty} r^{-\frac{1}{\alpha}}(s)\left(Q^{\sigma}(s)\right)^{1+\frac{1}{\alpha}} \Delta s \\
& =H^{\alpha}(t, \alpha), \quad t \in\left[t_{6}, \infty\right) .
\end{aligned}
$$

Since $r^{\frac{1}{\alpha}} y^{\Delta}$ is decreasing on $\left[t_{6}, \infty\right)$, we have

$$
\begin{aligned}
y(t)=y\left(t_{6}\right)+\int_{t_{6}}^{t} y^{\Delta}(s) \Delta s & =y\left(t_{6}\right)+\int_{t_{6}}^{t} r^{-\frac{1}{\alpha}}(s)\left(r^{\frac{1}{\alpha}}(s) y^{\Delta}(s)\right) \Delta s \\
& \geq r^{\frac{1}{\alpha}}(t) y^{\Delta}(t) \int_{t_{6}}^{t} r^{-\frac{1}{\alpha}}(s) \Delta s, \quad t \in\left[t_{6}, \infty\right) .
\end{aligned}
$$

Therefore, we obtain

$$
\frac{r^{\frac{1}{\alpha}}(t) y^{\Delta}(t)}{y(t)} \leq\left(\int_{t_{6}}^{t} r^{-\frac{1}{\alpha}}(s) \Delta s\right)^{-1}, \quad t \in\left[t_{6}, \infty\right)
$$

From (30), (19) and (31), we get

$$
H(t, \alpha) \leq w^{\frac{1}{\alpha}}(t)=\frac{r^{\frac{1}{\alpha}}(t) y^{\Delta}(t)}{y(t)} \leq\left(\int_{t_{6}}^{t} r^{-\frac{1}{\alpha}}(s) \Delta s\right)^{-1}, \quad t \in\left[t_{6}, \infty\right) .
$$

Thus, we find

$$
\left(\int_{t_{6}}^{t} r^{-\frac{1}{\alpha}}(s) \Delta s\right) H(t, \alpha) \leq 1, \quad t \in\left[t_{6}, \infty\right) .
$$

Taking lim sup of both sides of the last inequality as $t \rightarrow \infty$, we get a contradiction to (29). The proof is complete.

Theorem 3. Let $\beta<\alpha$, and suppose that (9) and (10) hold. If

$$
\limsup _{t \rightarrow \infty} Q^{(\alpha-\beta) /(\alpha \beta)}(t)\left(\int_{t_{0}}^{t} r^{-\frac{1}{\alpha}}(s) \Delta s\right)\left[Q(t)+c \int_{t}^{\infty} r^{-\frac{1}{\alpha}}(s)\left(Q^{\sigma}(s)\right)^{1+\frac{1}{\beta}} \Delta s\right]^{1 / \alpha}=\infty
$$

for every constant $c>0$, where Q is defined as in Theorem 1, then every bounded solution of (3) is oscillatory.

Proof. Suppose that $x$ is a bounded nonoscillatory solution of (3). Without loss of generality, we may assume that $x$ is a bounded eventually positive solution of (3). Proceeding as in the proof of Theorem 1 to obtain (12)-(16) and (25). It follows from (25) that $w(t) \geq Q(t)$ on $\left[t_{6}, \infty\right)$. Therefore, from (19) we have $r^{1 / \alpha} y^{\Delta} \geq y^{\beta / \alpha} Q^{1 / \alpha}$ on $\left[t_{6}, \infty\right)$. Since $r^{1 / \alpha} y^{\Delta}$ is a decreasing function on $\left[t_{6}, \infty\right)$, there exist $k>0$ and $t_{7} \geq t_{6}$ such that $k \geq r^{1 / \alpha} y^{\Delta} \geq y^{\beta / \alpha} Q^{1 / \alpha}$ on $\left[t_{7}, \infty\right)$. Thus, we get

$$
y \leq k^{\alpha / \beta} Q^{-1 / \beta} \text { on }\left[t_{7}, \infty\right) .
$$

Hence, we obtain

$$
\left(y^{\sigma}\right)^{(\beta-\alpha) / \alpha} \geq k^{(\beta-\alpha) / \beta}\left(Q^{\sigma}\right)^{(\alpha-\beta) /(\alpha \beta)} \text { on }\left[t_{7}, \infty\right) .
$$

Using (34) in (25) and noticing the definition of $w$, we see

$$
(y(t))^{(\alpha-\beta) / \alpha} \frac{r^{1 / \alpha}(t) y^{\Delta}(t)}{y(t)} \geq\left[Q(t)+c \int_{t}^{\infty} r^{-\frac{1}{\alpha}}(s)\left(Q^{\sigma}(s)\right)^{1+\frac{1}{\beta}} \Delta s\right]^{1 / \alpha}, \quad t \in\left[t_{7}, \infty\right),
$$

where $c:=\beta k^{(\beta-\alpha) / \beta}$. Using (31) and (33) in (35), we find

$$
k^{(\alpha-\beta) / \beta} Q^{(\beta-\alpha) /(\alpha \beta)}(t)\left(\int_{t_{6}}^{t} r^{-\frac{1}{\alpha}}(s) \Delta s\right)^{-1} \geq\left[Q(t)+c \int_{t}^{\infty} r^{-\frac{1}{\alpha}}(s)\left(Q^{\sigma}(s)\right)^{1+\frac{1}{\beta}} \Delta s\right]^{1 / \alpha}
$$


for $t \in\left[t_{7}, \infty\right)$. Therefore, we have

$$
k^{(\alpha-\beta) / \beta} \geq Q^{(\alpha-\beta) /(\alpha \beta)}(t)\left(\int_{t_{6}}^{t} r^{-\frac{1}{\alpha}}(s) \Delta s\right)\left[Q(t)+c \int_{t}^{\infty} r^{-\frac{1}{\alpha}}(s)\left(Q^{\sigma}(s)\right)^{1+\frac{1}{\beta}} \Delta s\right]^{1 / \alpha}
$$

for $t \in\left[t_{7}, \infty\right)$. Taking lim sup of both sides of the last inequality as $t \rightarrow \infty$, we get a contradiction to (32). The proof is complete.

\section{References}

Agarwal, R. P., Grace, S. R. \& O’Regan, D. (2003). Oscillation Theory for Second Order Dynamic Equations. London: Taylor \& Francis.

Bohner, M. \& Peterson, A. (2001). Dynamic Equations on Time Scales: An Introduction with Applications. Boston: Birkhäuser.

Bohner, M. \& Peterson, A. (2003). Advances in Dynamic Equations on Time Scales. Boston: Birkhäuser.

Chen, D. -X. \& Liu, J. -C. (2008). Asymptotic behavior and oscillation of solutions of third-order nonlinear neutral delay dynamic equations on time scales. Can. Appl. Math. Q., 16, 19-43.

Chen, D. -X. (2010). Oscillation and asymptotic behavior for $n$ th-order nonlinear neutral delay dynamic equations on time scales. Acta Appl. Math., 109, 703-719.

Erbe, L., Peterson, A. \& Saker, S. H. (2007). Hille and Nehari type criteria for third-order dynamic equations. J. Math. Anal. Appl., 329, 112-131.

Grace, S. R., Agarwal, R. P., Kaymakçalan, B. \& Sae-Jie, W. (2008). Oscillation criteria for second-order nonlinear dynamic equations. Can. Appl. Math. Q., 16, 59-76.

Grace, S. R., Agarwal, R. P., Kaymakçalan, B. \& Sae-Jie, W. (2009). On the oscillation of certain second order nonlinear dynamic equations. Math. Comput. Modelling, 50, 273-286.

Han, Z., Sun, S. \& Shi, B. (2007). Oscillation criteria for a class of second-order Emden-Fowler delay dynamic equations on time scales. J. Math. Anal. Appl., 334, 847-858.

Hassan, T. S. (2008). Oscillation criteria for half-linear dynamic equations on time scales. J. Math. Anal. Appl.. 345 , 176-185.

Medico, A. D. \& Kong, Q. (2004). Kamenev-type and interval oscillation criteria for second-order linear differential equations on a measure chain. J. Math. Anal. Appl., 294, 621-643.

Saker, S. H. (2005). Oscillation criteria of second-order half-linear dynamic equations on time scales. J. Comput. Appl. Math., 177, 375-387. 